$\xi=-1$

\title{
Path Optimization based on Bit Error Rate (BER) Estimation in Underwater Sensor Networks
}

\author{
S.Madhumita ${ }^{1^{*}}$, G.Niranjana ${ }^{2}$, V.M. Shenbagaraman ${ }^{3}$ \\ Department of Computer Science Engineering, \\ SRM Institute of Science and Technology, Kattankulathur-603203, India \\ *Corresponding Author E-mail: ' Imadhumita_2992@yahoo.com
}

\begin{abstract}
The underwater sensor networks (UWSNs) is an enabling technology and has become more and more trend for detecting vast area of oceans. Underwater sensor Networks consist of a variable number of sensors based on acoustic wireless communications. Communications are rapidly used for enabling advances in terms of ocean monitoring and observatory systems, deep sea surveillance and tracking of various entities of the aquatic environment and unearthing resources. But the drawback of the existing system is load and latency. Our proposed work mainly aimed to increase the throughput and optimize the path based upon relay node selection using BER estimation. And the performance of the system is analyzed using mathematical and simulation methods by comparing parameters such as Energy consumption, throughput, End to end delay.
\end{abstract}

IndexTerms: Underwater sensor networks (UWSNs), Bit Error Rate (BER), relay node.

\section{Introduction}

Our earth is covered by $75 \%$ of water that could be lake and ocean. Wireless information transmission through the ocean is one of the enabling technologies for the development of future oceanobservation systems and sensor networks. Applications of underwater sensing range from oil industry to aquaculture, and include instrument monitoring, pollution control, climate recording, prediction of natural disturbances, search and survey missions, and study of marine life.

Underwater sensor Networks consist of a variable number of sensors based on acoustic wireless communications. WSNs generally consist of a base station, which can communicate wirelessly with the sensor nodes. These sensor nodes collect data, compress it, and transmit it to the base station directly or indirectly through other sensor nodes present in the network. Anchor nodes are sensor nodes with known locations. This can be achieved by either equipping them with GPS or by carefully deploying them at known locations. Relay nodes are nodes which are intermediate between the source and destination

In this paper, we will be getting an optimized path between the source and destination based upon relay node selection using BER estimation. And the performance of the system using mathematical and simulation methods by comparing parameters such as Energy consumption, Throughput, End to end delay will be analyzed.

\section{Previous Work}

Severa researchers studied security challenges related to UWSNs. In this unit, we make a brief study of recent and relevant works Zhangbing Zhou, Wei Fang, JianweiNiu, Lei Shu, and Mithun
Mukherjee had proposed a Energy-Efficient Event Determination in Underwater WSNs Leveraging Practical Data Prediction.

They had transmitted data between the source and destination using relay nodes with remaining energy above the threshold value. And they have also considered the source nodes which has high data amount is sensed and passed to sink nodes in time slots. They proposed a practical data prediction mechanism for estimating sensory data and the geographical location of sensor nodes at sink nodes, and these data are synchronized with those sensed by underwater sensor nodes only when their variation is beyond a pre specified threshold. This approach is energyefficient, especially when the variation of network environments follows certain and simple patterns.

Underwater applications, like pollution monitoring and fish or microorganism tracking, depend on the monitoring of underwater environments, and they are supported currently by existingplatforms and infrastructure. Based on sensory data gathered at a certain time slot, the occurrence of a certain phenomenon (called an event) is detected with respect to certain criteria, and an appropriate response can be enacted accordingly. An example is the marine shellfish monitoring, where the properties including $\mathrm{pH}$ value, biotoxin value, water temperature, salinity, etc. are to be gathered and analyzed. When an event like a pollution is detected, sensory data about a certain set of properties should be collected and routed to sink node(s) immediately for the determination of event coverage and sources, which are applies for promoting the enactment of prompt and proper responses.

Itis worth noting that the occurrence of events typically account for a relatively small percentage of the network lifetime. The majority of sensor nodes may not need to route their sensory data to sink node(s) at most time durations, when these sensory data reflect the situation of healthy. In this setting, effective and energy-efficient techniques for the routing of sensory data and the determination of potential event coverage and sources, are fundamental. 
Event detection and event sources determination have been explored by recent works in underwater WSNs. Specifically, physical sensor nodes are abstracted and represented as virtual sensor nodes, which can be adopted for the localization of events, while keeping the sensing overhead relatively low.

Note that the cost for localizing physical sensor nodes may be quite high, since sensor nodes drift along with the water dynamics. Whereas a virtual sensor node is the representative of any physical sensor node located in a certain subregion, and hence,the event localization should be more accurate .

An event localization mechanism leveraging monitoring courses has been developed, where monitoring courses are adopted for the determination of event locations, and for the identification of potential network problems. Generally, these techniques are promising for event localisation in the underwater environment.

Note that an event may be reflected by the variation of sensory data of neighboring sensor nodes in a collective fashion, and the determination of potential event sources is critical for proper response enactment. In addition, sensory data may not vary dramatically especially when the network environment is relatively steady, which suggests that sensory data collected at previous time slots may be suitable to be reused for supporting certain applications in following time slots. Taking these factors into consideration, a cache-aware approach has been proposed to identify potential events leveraging sensory data of neighboring sensor nodes.

CDMA is an important thing for underwater acoustic networks. CDMA permits random access to shared communication channel. Its performance is robust to frequency selective fading caused by multipath transmission. Since this distinguishes among signals simultaneously which are transmitted by multiple devices through codes.

Existing work reduce the packet loss with the help of co operative sensing. Localization process is divided into two phases: 1. Transmission range and 2. Dynamic movement. During ranging, estimated sound speed and two ways message are used for distance measurement between sensor node and three neighboring anchor nodes. Error rate is avoided by using robustness condition for both surface plane and underwater plane. The disadvantages of this existing work are

(1)Speed and Velocity is time variance and difficult to find out.

(2)Time synchronization is difficult to achieve in underwater scenario due to long transmission delay and variable speed variance.

(3)Propagation delay is higher.

\section{Proposed Work}

\section{A. Network Simulator}

In network simulator is a software which is used for testing network components .This is easy to implement and can also be easily verified. This is a discrete event simulator.

It provides support for Simulation of TCP, Routing, Huge number of protocols ranging from wired to wireless networks, Supports the addition of new entities like agent, packet, application, queue, protocol, routing, etc.

NS2 is not a polished or a finished product .In this errors/Bugs are being discovered and corrected.

Network simulator architecture is said to consists of C++ (Internally), OTCL (User Interface) and TclCL (Interface between $\mathrm{C}++$ and OTCL).The simulation trace file of this consists of $\mathrm{NAM}$ ( animation) and X-graph

(plotting).

For UWSN there are tools such MANNASIM and Aquasim patch Mannasim and Aquasim package in ns2 Used to create a base Underwater wireless sensor Networks with more number of nodes and transfer the Packets from sensor node to receiver node.

\section{B. Architecture of UWSNs}

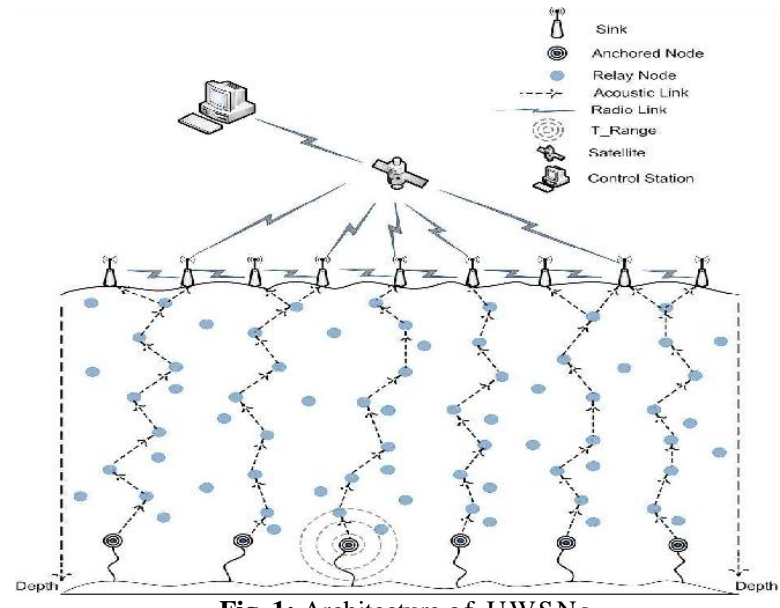

Fig. 1: Architecture of UWSNs

\section{Shortest Distance using Dijkstra's Algorithm}

Dijkstra's algorithm, published in 1959 and named after its creator Dutch computer scientist EdsgerDijkstra, can be applied on a weighted graph. The graph can either be directed or undirected. One stipulation to using the algorithm is that the graph needs to have a nonnegative weight on every edge.

We maintain two sets, one set contains vertices included in shortest path tree, other set includes vertices not yet included in shortest path tree. At every step of the algorithm, we find a vertex which is in the other set (set of not yet included) and has minimum distance from source.

\section{Dijkstra'sAlgorithm Example}

- Initialize the array smallestWeight so that smallestWeight[u] = weights[vertex, u].

- $\quad$ Set smallestWeight[vertex $]=0$.

- Find the vertex, $v$, that is closest to vertex for which the shortest path has not been determined.

- Mark v as the (next) vertex for which the smallest weight is found.

- For each vertex $w$ in $G$, such that the shortest path from vertex to $\mathrm{w}$ has not been determined and an edge $(\mathrm{v}, \mathrm{w})$ exists, if the weight of the path to $\mathrm{w}$ via $\mathrm{v}$ is smaller than its current weight, update the weight of $\mathrm{w}$ to the weight of $\mathrm{v}+$ the weight of the edge $(\mathrm{v}, \mathrm{w})$.

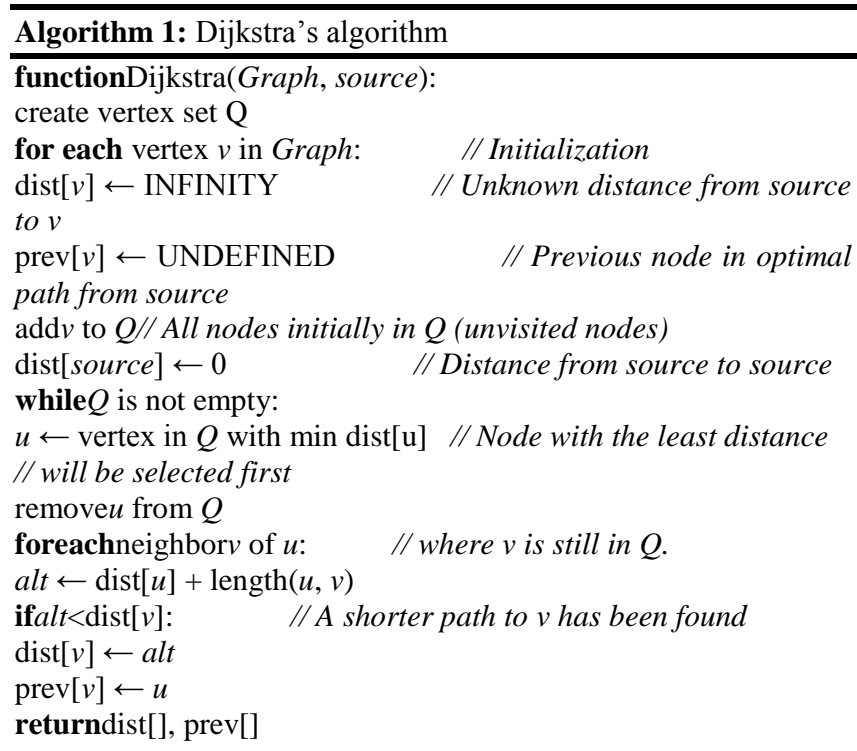


Using this algorithm two shortest path are found between the source and destination.

\section{BER Calculation}

We need to calculate $\mathrm{Eb} / \mathrm{N} 0$ (the energy per bit to noise power spectral density ratio) which is an important parameter in digital communication or data transmission. It is a normalized signal-tonoise ratio (SNR) measure, also known as the "SNR per bit". It is especially useful when comparing the bit error rate (BER) performance.

The source node sends data to the relay node which has less Bit Error Rate between the two shortest paths calculated.This process is repeated till the data reaches the destination.Thus,data is transmitted between the source and destination with less packet loss.

As an example, assume this transmitted bit sequence:

0110001011

and the following received bit sequence:

0010101001 ,

The number of bit errors (the underlined bits) is, in this case, 3 . The BER is 3 incorrect bits divided by 10 transferred bits, resulting in a BER of 0.3 or $30 \%$.

In a communication system, the receiver side BER may be affected by transmission channel noise, interference, distortion, bit synchronization problems, attenuation, wireless multipath fading, etc.The higher(Better) the SNR the Better the BER.

Short for signal-to-noise ratio, the ratio of the amplitude of a desired analog or digital data signal to the amplitude of noise in a transmission channel at a specific point in time. SNR is typically expressed logarithmically in decibels (dB).

SNR measures the quality of a transmission channel or an audio signal over a network channel. The greater the ratio, the easier it is to identify and subsequently isolate and eliminate the source of noise. A SNR of zero indicates that the desired signal is virtually indistinguishable from the unwanted noise

The usual procedure to calculate this is:

1) The nodes positions are known

2) The channel model is also known

3) The antenna model, the antenna characteristics, the Tx power, the Rx noise figure and so on are also known.

4) Now we can calculate the signal and the noise power at the receiver (there may be a random factor to consider the model randomness).

5) Then we can calculate the BER

Algorithm 2: For BER (Bit Error Rate)

Step1: Selection of relay nodes based upon SINR (Signal Interference Noise Ratio) ratio.

$$
\operatorname{SINR}=\frac{\hat{\mathrm{N}}_{r p}}{\hat{\mathrm{N}}_{n i}}
$$

Where

SINR $=($ Signal Interference Noise Ratio $)$ ratio.

$\hat{\mathrm{N}}_{r p}=$ Total power of the node across the bandwidth.

$\hat{\mathrm{N}}_{n i}=$ Total noise interference received by node across the bandwidth.

Step2: Path selection based on DIJKSTRA shortest path selection.

$$
\begin{aligned}
& \bigoplus_{\mathrm{i}}=\mathrm{P}_{\mathbf{1 i}} \\
& i=1,2 \ldots n \\
& \breve{k}=1,2,3 \ldots n-1 \\
& \text { For all point of } \mathbf{T} \\
& \bigoplus_{2}=\min \left\{\bigoplus_{2}, \bigoplus_{u}+\mathrm{P}_{u, 2}\right\} \text {, } \\
& \mathrm{Ð}_{3}=\min \left\{\mathrm{Ð}_{3}, \bigoplus_{u}+\mathrm{P}_{u, 3}\right\} \text {, } \\
& \bigoplus_{\mathbf{\eta}}=\min \left\{\bigoplus_{\mathfrak{\eta}}, \oplus_{u}+P_{u, \eta}\right\} \text {, }
\end{aligned}
$$

Where,

$\bigoplus_{\mathrm{i}}=$ distance between two nodes.

$\mathrm{P}_{1 \mathrm{i}}=$ position of nodes

Step3: Selection of relay nodes from source to destination.
Step4: Calculating the Fitness value of relay nodes.

$v=1,2 \ldots \eta$

$$
\mathrm{F}_{v}=\gamma \mathrm{f}
$$

Where

$\gamma=$ coefficient of energy consumption.

$$
f=\frac{\sum_{i=1}^{N} \check{E}\left(\eta_{i}\right)}{\sum_{\grave{k}=1}^{\grave{E}} \check{E}\left(P_{i}\right)}
$$

$\eta_{\mathrm{i}}=$ initial energy of nodes

$\mathrm{P}_{\mathrm{i}}=$ Position of nodes.

Step5: Selection of forwarder node based on fitness value.

$$
\text { If }\left(\mathrm{F}_{v}<\mathrm{F}_{v R}\right)\{
$$

Return $\mathrm{F}_{v R}$ is forwarder node.

\}

Else

\{

Return $\mathrm{F}_{v}$ is forwarder node.

\}

Step6: Transmitting the data from forwarder node to destination.Using this we can calculate the BER for each relay node and we can select the relay node with least BER to transmit data from source to destination.

\section{E. Block Diagram}

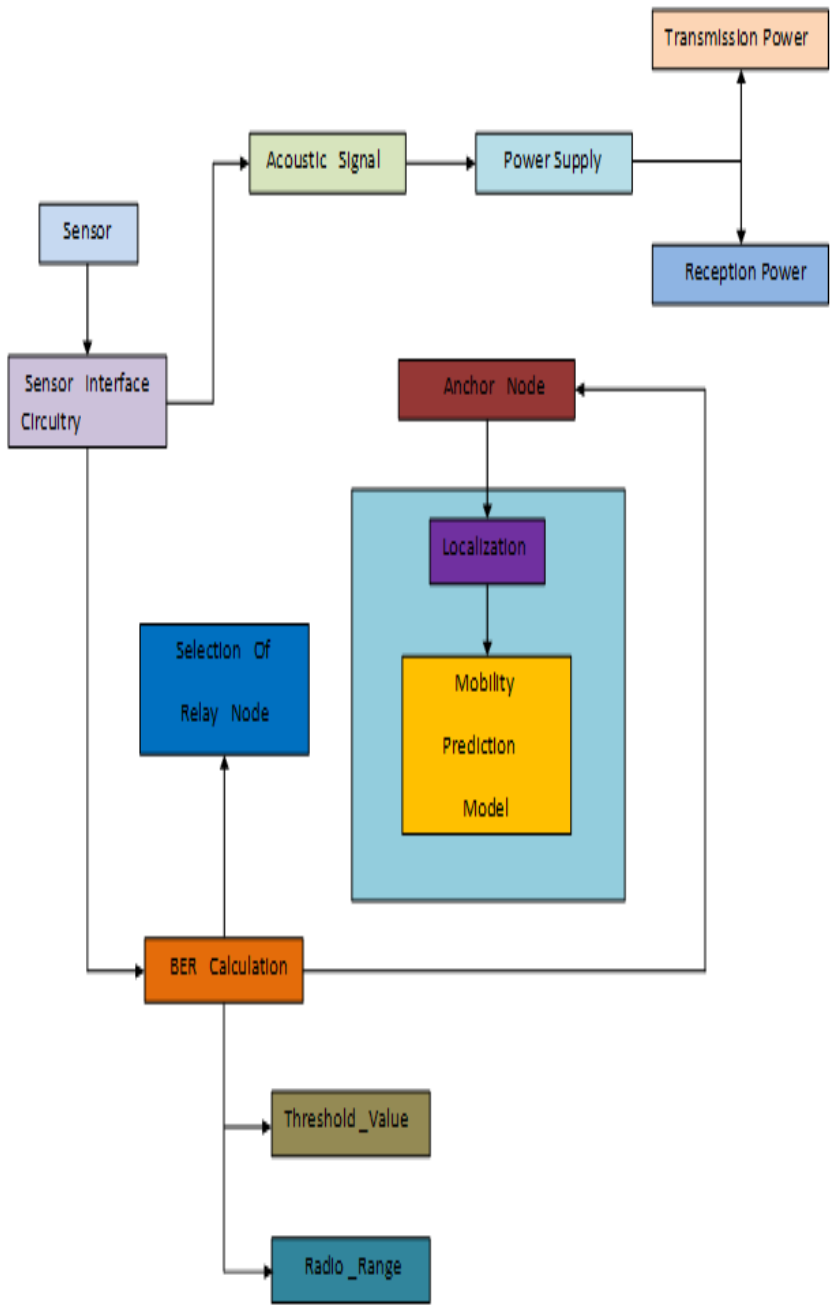

Fig. 2: Block Diagram of UWSNs

In this proposed work, anchor nodes will be based on localization technique and mobility prediction model. The relay nodes will be selected based on BER calculation .The nodes which are within the radio range and above the threshold value will be selected and data will be transmitted between source and destination. 


\section{F. Flow Chart}

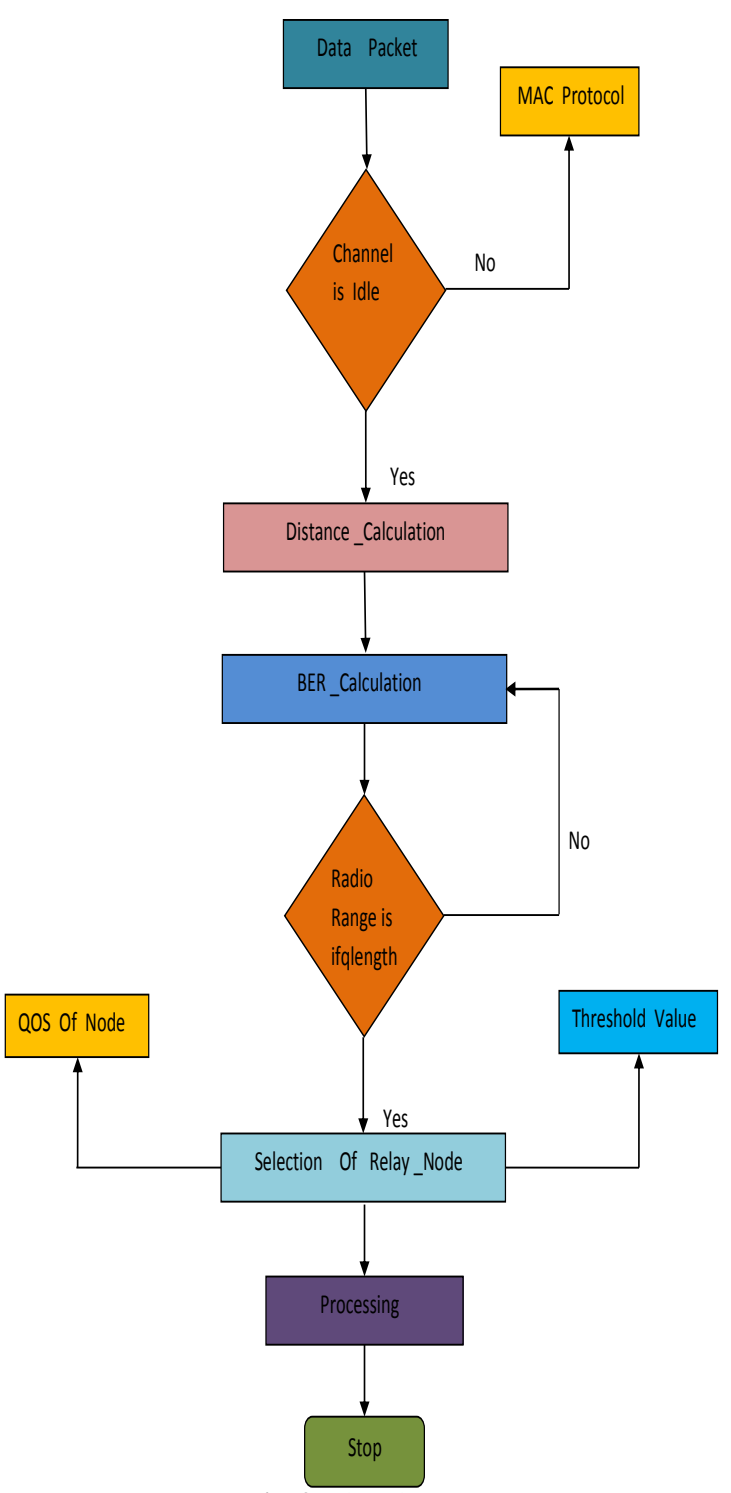

Fig. 3: Flowchart

\section{G. Results}

(a) Simulation_Time Vs Throughput

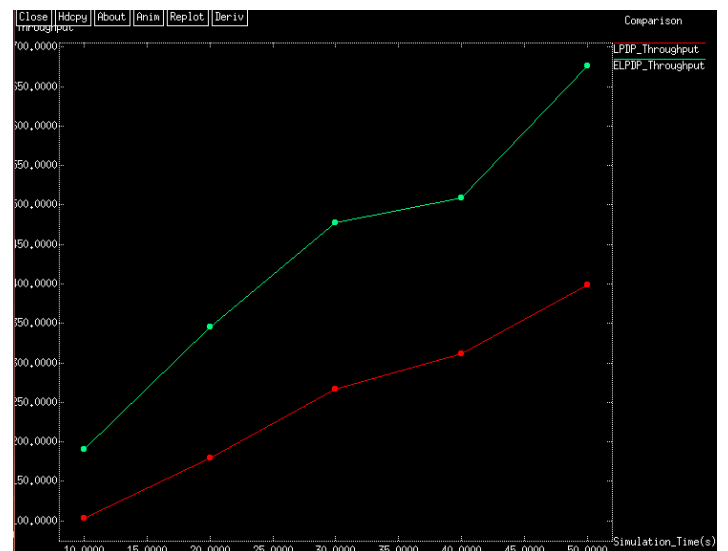

Fig. 4: Existing system Vs prosposed system (Throughput)

Throughput has been considerably increased when compared to the existing system as data is transmitted via relay nodes which has less Bit error rate in the transmission range. (b) Simulation_TimeVs Energy

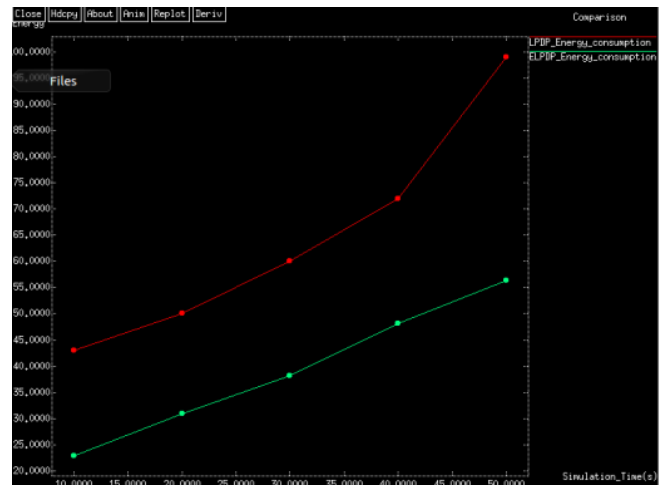

Fig. 5: Existing system Vs prosposed system (Energy)

Energy consumed by the nodes is reduced by $20 \%$ when compared to the existing system.

\section{(c) Simulation time Vs BER}

\begin{tabular}{|r|r|r|r|}
\hline S.NO SIMULATION TIME EXISTING SYSTEM PROPOSED SYSTEM \\
\hline & & BIT ERROR RATE & BIT ERROR RATE \\
\hline 1 & 10 & 0.32 & 0.23 \\
\hline 2 & 15 & 0.31 & 0.27 \\
\hline 3 & 20 & 0.36 & 0.3 \\
\hline 4 & 25 & 0.42 & 0.32 \\
\hline 5 & 30 & 0.48 & 0.35 \\
\hline
\end{tabular}

In the existing system,data is transmitted between source and destination via relay nodes which has higher Bit Error Rate when compared the proposed system.

\section{Conclusion}

Hence in this paper we have proposed an optimization path between source and destination based on relay node selection using BER calculation. The results of our proposed technique will ensure less packet loss .Energy consumption, throughput, End to end delay will be calculated and outputs will be shown using graphs. The Performance will be analyzed based on these parameters using mathematical and simulation means.

\section{Reference}

[1] Zhangbing Zhou, Wei Fang, JianweiNiu, Lei Shu, and Mithun Mukherjee, "Energy-Efficient Event Determination in Underwater WSNs Leveraging Practical Data Prediction",IEEE TRANSACTIONS ON INDUSTRIAL INFORMATICS, VOL. 13 , NO. 3, JUNE 2017

[2] N. Li, J.-F. Martinez, J. M. M. Chaus, and M. Eckert, "A survey on underwater acoustic sensor network routing protocols," Sensors, vol. 16, no. 3, pp. 1-28, 2016.

[3] Luca Ghelardoni,AlessandroGhio,DavideAnguita, "Smart underwater Wireless Sensor Networks," IEEE Convention of Electrical and Electronics Engineers in Israel.,2012.

[4] Yuri Labrador, MasoumehKarimi , Deng Pan, and Jerry MillerWORK, "Modulation and Error Correction in the Underwater Acoustic Communication Channel", IJCSNS International Journal of Computer Science and Network Security, VOL.9 No.7, July 2009 